\title{
ACCRETION DISK WINDS IN CATACLYSMIC VARIABLES
}

\author{
CHRISTIAN KNIGGE ${ }^{1}$, JANET E. DREW ${ }^{1}$, \\ KEITH O. MASON ${ }^{2}$ \\ 1. Nucl. \& Astroph. Lab., Keble Road, Oxford, OX1 3RH, UK \\ 2. Mullard Spa. Sci. Lab., Surrey, RH5 6NT, UK
}

\begin{abstract}
We present preliminary results of a modelling campaign based on HST eclipse observations of the wind-formed $\mathrm{ClV}$ resonance line in the eclipsing nova-like variable UX UMa. Within the framework of a simple kinematic accretion disk wind model, we are currently able to reproduce the shapes (but not the strengths) of the observed line profiles away from and during eclipse, as well as the behaviour of the integrated line flux light curve. The most important aspect of our modelling is that it suggests the presence of a vertically extended, relatively dense and slow moving transition region between the disk photosphere and the fast wind.
\end{abstract}

\section{Introduction}

Eclipse observations of the wind-formed UV resonance lines in high inclination CVs contain a wealth of information about the kinematics and geometry of the line forming region. Detailed modelling of such data - which can only be gathered with the Hubble Space Telescope (HST) - should produce a much improved physical picture of $\mathrm{CV}$ winds. Here we present preliminary results of the first modelling campaign of this kind, in which we are able to draw on HST eclipse observations of the C IV wind line in the nova-like variable UX UMa (Mason et al. 1995).

\section{Model framework}

The kinematic accretion disk wind model we use is that of Knigge, Woods \& Drew (1995) and is illustrated in Fig. 1. In it, all streamlines converge at a fiducial point a distance, $d$, above/below the disk, which allows the geometric outflow collimation to be controlled by this single free parameter. A simple power law scaling with the local disk temperature is used to fix the local mass-loss rate per unit area from the disk: $\dot{m} \propto T_{\text {disk }}(R)^{4 \alpha}$. 


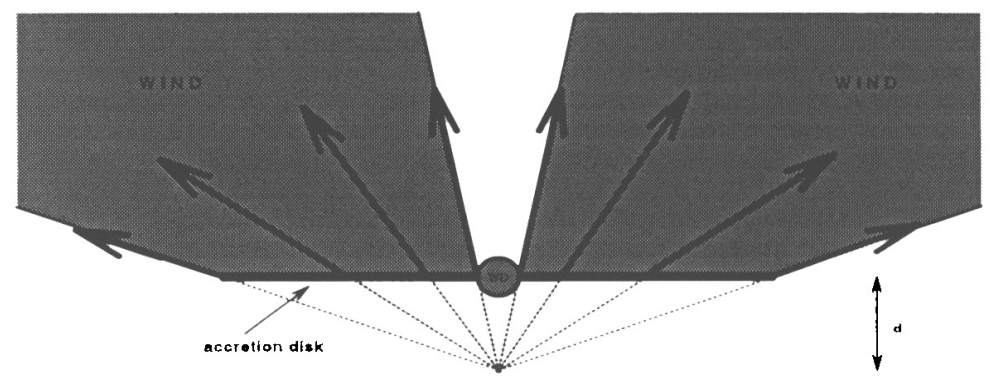

Figure 1 . The geometry of our kinematic disk wind model.

The wind is assumed to rotate initially with the disk's Keplerian velocity and to conserve its specific angular momentum as it rises above the binary plane. For the non-rotational part of the wind velocity field we use a description which is similar in form to the $\beta$-type velocity law often used in models of hot star winds (Castor \& Lamers 1979).

A three-dimensional Monte Carlo line transfer code is used to synthesize the line profile arising from our accretion disk wind models (Knigge et al. 1995). This ensures that multiple scattering in the outflow is treated selfconsistently in these calculations.

The continuum light is assumed to be produced by a steady-state accretion disk with $\dot{M}_{\mathrm{acc}} \simeq 310^{-8} \mathrm{M}_{\odot \mathrm{yr}^{-1}}$ and a hot white dwarf (WD). The latter contributes about $20 \%$ to UX UMa's out-of-eclipse continuum flux, which corresponds to $T_{\mathrm{WD}} \simeq 8.510^{4} \mathrm{~K}$. The fit to the observed continuum light curve achieved by this simple model is shown in the left panel of Fig. 2. Here and throughout, Baptista et al.'s (1995) system parameters for UX UMa (most importantly $i=71^{\circ}$ and $q=1$ ) have been used.

\section{Preliminary results}

Fig. 2 shows the light curves of UX UMa's UV continuum and integrated $\mathrm{C}$ IV line flux. Note that the shapes of the two light curves are radically different, with the net line flux exhibiting an increase above the out-ofeclipse level at phases close to mid-eclipse.

Some more insight can be gained by comparing the appearance of the C IV line around $\phi_{\text {orb }} \simeq 0.9$ with that of the profile near $\phi_{\text {orb }} \simeq 0$ (Fig. 3 ). The most striking aspect of this comparison is the presence of two absorption reversals close to the rest wavelengths of the resonance doublet in the pre-eclipse profile, which disappear during the eclipse to reveal a simpler, if asymmetric emission line.

The results of line profile syntheses for our current 'best-bet' model are also illustrated in Figs. 2 and 3. These show that the shapes of the 

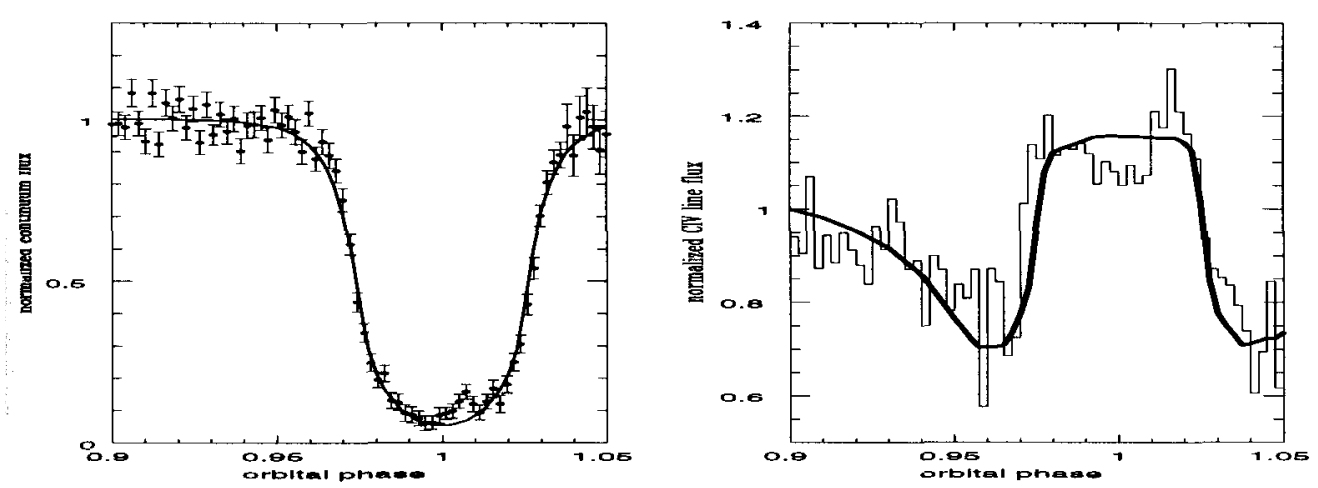

Figure 2. Left panel: UX UMa's normalised UV continuum light curve (points) and the fit achieved by our steady-state disk + hot WD model (solid line). Right Panel: The normalised CIV continuum-subtracted, integrated line flux light curve (histogram) and the fit achieved by our current best-bet accretion disk wind model (continuous line).

profiles and the line flux light curve are reproduced reasonably well, but that the model still underestimates the overall emission strength. A possible explanation for this failure is offered below.

Assuming that our modelling does reflect reality to some extent, what is the picture that emerges of the outflow in UX UMa?

First of all, its geometric collimation seems to be relatively well constrained by the data, with indicated inner and outer wind cone opening angles of about $5^{\circ}$ and $65^{\circ}\left(d \simeq 15 R_{W D}\right)$. The mass-loss power law index, $\alpha$, on the other hand, has less influence on the emergent line profiles; in the model shown here it has been set equal to unity. Moreover, the wind acceleration beyond the sonic point (whose position in the outflow we will return to below) seems to be fairly gradual, with a velocity law index of $\beta=4.5$ describing our current best model. The effective mass-loss rate inferred from our modelling (which is approximately equal to the true mass-loss rate if C IV is the dominant ionization stage in the outflow) is $\dot{M}_{\text {eff }} \sim 0.01 \dot{M}_{\text {acc }}$

However, our most important finding thus far is that in order to reproduce the shape and eclipse behaviour of the absorption reversals in the observational line profiles, it is necessary to invoke the presence of a vertically extended, relatively dense and slow moving outflow region just above the accretion disk (which we model by moving the position of the sonic point of the wind away from the disk surface). This is not unreasonable, since such a region (i) can produce strong absorption effects by virtue of its high density, (ii) will absorb photons primarily at low velocities and thus close to line centre, (iii) lies close enough to the disk plane to be eclipsed by the secondary.

While alternative scenarios for the origin of the reversals - such as absorption in some sort of disk edge structure - cannot yet be ruled out, 

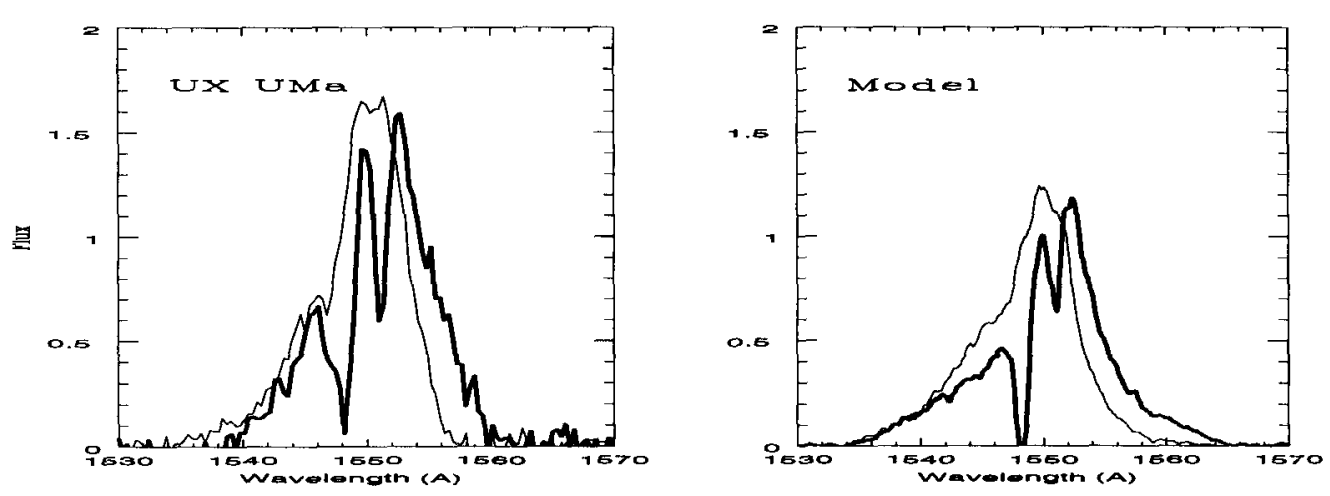

Figure 3. Left panel: UX UMa's average pre- (heavy line) and mid-eclipse (thin line) CIV line profiles. Right Panel: Pre- (heavy line) and mid-eclipse (thin line) CIV line profiles arising from our accretion disk wind model. All profiles have been continuum subtracted and normalised to a common scale.

our preferred interpretation is as evidence for the existence of a transition region between the disk photosphere and the fast moving outflow. This is in line with a recent analytical study of line-driven winds from accretion disks, in which the height of the sonic point above the disk plane was predicted to scale roughly as $z_{s} \sim R$ (Suleimanov 1995). [Our modelling of the position of the sonic point in the outflow is in fact loosely based on this scaling.] Our findings may also be related to the absorbing Fe-curtain which veils the WD in the dwarf nova OY Car during quiescence (Horne et al. 1994).

We finally note that, due to the high densities occuring in this transition region, line photons tend to scatter many times in our model before escaping. This means that the use of a Monte Carlo radiative transfer code is certainly warranted here. It also means, however, that the conservative scattering approximation we currently make in our calculations may not be sufficiently accurate. If thermal wind emission contributes significantly to the observed CIV line flux, this may help to explain the relative weakness of the model line profiles. This possibility is currently under examination.

\section{References}

Baptista, R., Horne, K., Hilditch, R.W., et al., 1995, Ap. J., 448, 395

Castor, J.I., Lamers, H.J.G.L.M, 1979, Ap. J. Supp., 39, 481

Horne, K., Marsh T.R., Cheng, F.H., et al., 1994, Ap. J., 426, 294

Knigge, C., Woods, J.A., Drew, J.E., 1995 MNRAS, 273, 225

Mason, K.O., Drew, J.E., Córdova, F.A., et al., 1995, MNRAS, 274, 271

Suleimanov, V.F., 1995, Astr. Lett., 21, 126 